\title{
Soybean Plant Disease Classification using Archimedes Optimization Algorithm based Hybrid Deep Learning Model
}

\section{J. Annrose ( $\boldsymbol{\nabla}$ jannrose.id@gmail.com )}

Anna University Chennai

\section{N. Herald Anantha Rufus}

Vel Tech Dr RR and Dr SR Technical University: Vel Tech Rangarajan Dr Sagunthala R\&amp;D Institute of Science and Technology

C. R. Edwin Selva Rex

Vignana Bharathi Institute of Technology

D. Godwin Immanuel

Sathyabama Institute of Science and Technology

\section{Research Article}

Keywords: Bean disease classification, Hybrid deep learning, Archimedes optimization algorithm, LSTM, and WPD

Posted Date: March 16th, 2021

DOI: https://doi.org/10.21203/rs.3.rs-281525/v1

License: (c) (i) This work is licensed under a Creative Commons Attribution 4.0 International License. Read Full License 


\title{
Soybean Plant Disease Classification using Archimedes Optimization
}

\section{Algorithm based Hybrid Deep Learning Model}

\author{
${ }^{1 *}$ Dr. J. Annrose, ${ }^{2}$ Dr. N. Herald Anantha Rufus, ${ }^{3}$ Dr. C. R. Edwin Selva Rex, \\ ${ }^{4}$ Dr. D. Godwin Immanuel \\ ${ }^{1}$ Assistant Professor, Department of Information Technology \\ St. Xavier's Catholic College of Engineering, Anna University, India. \\ ${ }^{2}$ Associate Professor, Department of Electronics and Communication Engineering, \\ Vel Tech Rangarajan Dr. Sagunthala $R$ \& D Institute of Science and Technology, Chennai. \\ ${ }^{3}$ Associate Professor, Department of Electrical and Electronics Engineering \\ Vignana Bharathi Institute of Technology, Hyderabad, India. \\ ${ }^{4}$ Associate Professor, Department of Electrical and Electronics Engineering \\ Sathyabama Institute of Science and Technology, Chennai, India. \\ *.Corresponding Author Email: jannrose.id@gmail.com
}

\begin{abstract}
:
Bean which is botanically called Phaseolus vulgaris L belongs to the Fabaceae family.During bean disease identification, unnecessary economical losses occur due to the delay of the treatment period, incorrect treatment, and lack of knowledge. The existing deep learning and machine learning techniques met few issues such as high computational complexity, higher cost associated with the training data, more execution time, noise, feature dimensionality, lower accuracy, low speed, etc. To tackle these problems, we have proposed a hybrid deep learning model with an Archimedes optimization algorithm (HDL-AOA) for bean disease classification. In this work, there are five bean classes of which one is a healthy class whereas the remaining four classes indicate different diseases such as Bean halo blight, Pythium diseases, Rhizoctonia root rot, and Anthracnose abnormalities acquired from the Soybean (Large) Data Set.The hybrid deep learning technique is the combination of wavelet packet decomposition (WPD) and long short term memory (LSTM). Initially, the WPD decomposes the input images into four subseries. For these sub-series, four LSTM networks were developed. During bean disease classification, an Archimedes optimization algorithm (AOA) enhances the classification accuracy for multiple single LSTM networks. MATLAB software implements the HDL-AOA model for bean disease classification. The proposed model accomplishes lower MAPE than other exiting methods. Finally, the proposed HDL-AOA model outperforms excellent classification results using different evaluation measures such as accuracy, specificity, sensitivity, precision, recall, and F-score.
\end{abstract}

Keywords: Bean disease classification; Hybrid deep learning; Archimedes optimization algorithm; LSTM; and WPD. 


\section{Introduction:}

Agriculture is one of the major pillars in the economic sector that contribute toincome, employment generation, and wealth for the country. Plant disease is the major factor to degrade agricultural products all over the world that leads to huge loss each year. The most produced and most important legumein the world is the bean (Phaseolus vulgaris L). On a global scale, the bean is an important legume/grain taken for consumption and was widely dispersed due to its broad adaptation [1,28]. During the growing season, the plants grow well in about $400 \mathrm{~mm}$ of precipitation and moderate growing temperatures $\left(>10{ }^{\circ} \mathrm{C}\right.$ and $\left.<30{ }^{\circ} \mathrm{C}\right)$. The growing temperatures $<30{ }^{\circ} \mathrm{C}$ with the tropical highlands and 60-120 days of frost-free growth are permitted by season where the ordinary bean is grown ina moderate growing region [2]. Approximately, $15 \%$ soluble fiber, $62 \%$ complex carbohydrates, several micronutrients $(\mathrm{Ca}, \mathrm{Fe}$, $\mathrm{Mg}, \mathrm{P}$, and $\mathrm{K}$ ), and $22 \%$ protein present in the uncooked dry bean. Bean commonly provides trace nutrients, calories, and sources of protein to persons who never give additional expensive sources of nutrition. The dry edible beans are a major kind in contemporary agriculture in which the matured seed is prepared in main dishes and harvested.

Root diseases (such as Fusarium root rot, Pythium diseases, Rhizoctonia root rot, Soybean cyst nematode, and Soybean cyst nematode soil sampling), Stem and wilt diseases (including Bacterial wilt, Fusarium yellows, stem rot, and white mold), and Foliar diseases such as (Anthracnose, Bacterial brown spot, Bean common mosaic, Common bean root dust, common bacterial blight, and halo blight) are the important types of bean diseases [3, 29]. Bean disease identification and detection at an early stage is a more important one. Hence, the people quickly take suitable prevention criteria. According to the experience in observing bean disease identification, most forest producers judge a degree and disease species in traditional forestry and agricultural production in which it needs skill and knowledge to detect the symptoms of bean diseases. The unnecessary economical losses are resultant due to delay of the treatment period, incorrect treatment, and lack of knowledge to plant disease identification. It will take little time even if experts are invited to identify a disease [4]. Hence,the automatic implementation of plant diseases or bean disease identification and classification is essential [30, 31].

More computer vision methods were applied to plant disease classification with the development of computational systems in recent years. Nowadays, artificial intelligence and data mining techniques are widely used to solve the prediction and classification issues in agriculture [5]. The execution measurements and 10 fold cross-validation with Decision Tree (DT), kNearest Neighbors (kNN), Backing Vector Machine (SVM), and Multilayer perceptron (MLP) models were used [6]. Deep learning techniques play a vital role in plant disease classification. These machine learning and deep learning techniques met few shortcomings in terms of computational complexity, the high cost associated with training data, more execution time, noise, feature dimensionality, lower accuracy, low speed, etc [7-10]. To tackle these issues, we have proposed a hybrid deep learning model with an Archimedes optimization algorithm (HDLAOA). The major contribution of this paper is summarized as follows: 
* The combination of wavelet packet decomposition with long short-term memory is proposed as a hybrid deep learning model.

* The classification accuracy for multiple single LSTM networks during bean disease classification is boosted up using the Archimedes optimization algorithm (AOA).

* The healthy and unhealthy classes such as Bean halo blight, Pythium diseases, Rhizoctonia root rot, and Anthracnose images were collected from Soybean (Large) Data Set.

The rest of the paper is organized as: Various plant disease classifications based on deep learning techniques are delineated in section 2. Section 3 explains the proposed HDL-AOA model for bean disease classification. The experimental investigation based on the proposed model is discussed in section 4. Finally, section 5 concludes the paper.

\section{Related work:}

This section reviews a few of the existing works based on plant disease classification using deep learning techniques.A deep convolutional neural network (DCNN) was proposed by Uğuz et al. [7] for olive peacock spot and aculeus olearius diseases classification. There are 3400 olive leaves sampleschosen in which three classes namely healthy, olive peacock spot and aculeus olearius disease were present. The VGG16 and VGG19 architectures based on the DCNN model are used. The network's performance was improved with the help of the RMS Prop optimization algorithm and Stochastic gradient descent. The multi-task system based on Convolutional Neural Networks (CNN) was suggested by Esgario et al. [8] to detect the stress severity caused by biotic agents on coffee leaves. A more accurate and robust detection system was performed using computational experiments. As a result of $86.51 \%$ severity estimation and $95.24 \%$ of biotic stress classification accuracy were obtained using multi-task system-based CNN with ResNet50 architecture. Hence, this method is appropriate for biotic stresses in coffee plantation identification and quantification.

A pre-trained Convolutional Neural Network (CNN) was proposed by Esgario et al. [9] for crop disease identification. This pre-trained CNN includes models such as DenseNet201, ResNet101, GoogLeNet, VGG19, Visual Geometry Group 16 (VGG16), and AlexNet. The author chose four kinds of crops with ten diseases such as leafhopper, yellow vein mosaic virus, citrus canker, citrus Hindu mite, brown spot, Cercospora leaf spot, two-spotted spider mite, Epilachna beetle, little leaf disease, and Tobacco Mosaic Virus TMV. For each disease class, they evaluated the prediction scores and classified images in real-time.Hernández et al. [10] proposed a Bayesian Deep Learning technique for the detection of plant diseases. When compared to the state of art methods, higher classification performance is achieved using Bayesian inference. The uncertainty of the predictions is quantified and posterior density for the plant disease detection problem was approximated. 
Table 1: Literature analysis based on plant disease classification using deep learning techniques

\begin{tabular}{|c|c|c|c|c|}
\hline Author & $\begin{array}{l}\text { Techniques } \\
\text { used }\end{array}$ & Name of the diseases & Advantages & Limitations \\
\hline $\begin{array}{l}\text { Uğuz et al. } \\
\text { [7] }\end{array}$ & DCNN & $\begin{array}{l}\text { Olive peacock spot and } \\
\text { Aculusolearius diseases }\end{array}$ & $\begin{array}{l}\text { Higher accuracy } \\
\text { ratings }\end{array}$ & $\begin{array}{c}\text { Higher } \\
\text { computational } \\
\text { complexities }\end{array}$ \\
\hline $\begin{array}{l}\text { Esgario et } \\
\text { al. [8] }\end{array}$ & $\begin{array}{l}\text { The multi-task } \\
\text { system based } \\
\text { CNN }\end{array}$ & $\begin{array}{c}\text { Cercospora leaf, brown } \\
\text { leaf spot, rust, and spot } \\
\text { leaf miner in coffee } \\
\text { leaves }\end{array}$ & $\begin{array}{c}\text { Accuracy of } \\
86.51 \% \text { severity } \\
\text { estimation and } \\
95.24 \% \text { biotic } \\
\text { stress } \\
\text { classification }\end{array}$ & $\begin{array}{c}\text { Low } \\
\text { representativity } \\
\text { of the dataset }\end{array}$ \\
\hline $\begin{array}{c}\text { Esgario et } \\
\text { al. [9] }\end{array}$ & $\begin{array}{l}\text { Pre-trained } \\
\text { CNN }\end{array}$ & $\begin{array}{l}\text { Leafhopper, yellow } \\
\text { vein mosaic virus, } \\
\text { citrus canker, citrus } \\
\text { Hindu mite, brown } \\
\text { spot, Cercospora leaf } \\
\text { spot, two-spotted spider } \\
\text { mite, Epilachna beetle, } \\
\text { little leaf disease, and } \\
\text { TMV }\end{array}$ & $\begin{array}{c}97.3 \% \text { best } \\
\text { validation } \\
\text { accuracy using } \\
\text { GoogLeNetand } \\
\text { cost-effective }\end{array}$ & $\begin{array}{l}\text { Takes higher } \\
\text { execution time }\end{array}$ \\
\hline $\begin{array}{c}\text { Hernández } \\
\text { et al. [10] }\end{array}$ & $\begin{array}{l}\text { Bayesian Deep } \\
\text { Learning }\end{array}$ & $\begin{array}{c}\text { Grape black spot and } \\
\text { grape escass }\end{array}$ & $\begin{array}{c}\text { Correct class } \\
\text { prediction results }\end{array}$ & $\begin{array}{c}\text { Data overfitting } \\
\text { issues }\end{array}$ \\
\hline $\begin{array}{c}\text { Chen et al. } \\
\text { [11] }\end{array}$ & DCNN & Maize and rice diseases & $\begin{array}{l}\text { The global } \\
\text { pooling layer } \\
\text { reduced the } \\
\text { dimension of } \\
\text { feature maps }\end{array}$ & $\begin{array}{l}\text { Higher- } \\
\text { dimensional } \\
\text { features }\end{array}$ \\
\hline $\begin{array}{l}\text { Li et al. } \\
{[12]}\end{array}$ & $\mathrm{CNN}$ & Ginkgo leaf disease & $\begin{array}{c}\text { Accomplished } \\
98.44 \% \text { accuracy } \\
\text { under laboratory } \\
\text { conditions }\end{array}$ & $\begin{array}{l}\text { Data overfitting } \\
\text { with more } \\
\text { processing time }\end{array}$ \\
\hline
\end{tabular}

The transfer learning of the deep convolutional neural networks (DCNN) was introduced by Chen et al. [11] for plant leaf disease identification. They selected the Inception module and VGGNet pre-trained on ImageNet for experimental investigation. According to the experimental investigation, $92.00 \%$ classification accuracy is obtained for rice plant images. For classification, the high-dimensional features are extracted and the experimental results provided better results in terms of both own dataset and public dataset. Finally, 91.83\% validation accuracy is achieved during disease classification. Li et al. [12] proposed Convolutional Neural Network (CNN) for Ginkgo leaf disease detection. They combined and used Inception V3 and VGGNet- 16 models. Based on laboratory conditions, $98.44 \%$ accuracy was accomplished and the VGG model provided $92.19 \%$ accuracy. Nevertheless, data overfitting with more processing time takes place. The existing work based on the plant disease classification using deep learning techniques is delineated in Table 1. 


\section{Hybrid deep learning with AOA model for bean disease classification:}

In this section, we have proposed hybrid deep learning with AOA (HDL-AOA) model forthe bean disease classification. For this work, the normal healthybean with four unhealthy classes such as Bean halo blight, Pythium diseases, Rhizoctonia root rot, Anthracnose were chosen. The steps involved in the proposed HDL-AOA model for bean disease classification are briefly explained in the following section.

\subsection{Wavelet Packet Decomposition:}

The time-frequency localization function with orthogonal wavelet packet bases and image properties are analyzed using an efficient tool called Wavelet Packet Decomposition (WPD). The original images are decomposed into sub-layers in which WPD is a kind of wavelet decomposition (WD). The low pass components of frequencies are segregated [13]. The timefrequency planes more than wavelet decompositionare divided and WPD accomplished a more precise analysis of images. When compared to WD, the WPD is more efficient for singular components, instantaneous, unbalanced, and identifying weak images. The WPD decomposes a coefficient of each layer continually. Both discrete and continuous transform are present in the WPD. The mother wavelet transform function $\phi(t)$ adopts the continuous wavelet transform for a signal $F(t)[14]$.

$$
C W T_{F}(a, b)=\left\langle F(t), \phi_{c, d}(t)\right\rangle=\int_{-\infty}^{\infty} F(t) \phi *((t-d) / c) \sqrt{c} d t
$$

The scale coefficient and translation coefficient are denoted as $c$ and $d$. The complex conjugates are denoted as $*$. The following equation describes a WPD process of decomposition.

$$
\left\{\begin{array}{l}
Q_{j}^{2 k-1}(t)=P H_{j-1}^{k}(t) \\
Q_{j}^{2 k}(t)=P G_{j-1}^{k}(t)
\end{array}\right.
$$

Equation (3) describes the reconstruction process of WPD.

$$
Q_{j}^{k}(t)=H * Q_{j+1}^{2 j-1}(t)+G * Q_{j+1}^{2 k}(t)
$$

Therefore, the time index and the wavelet packet levels are denoted as $t$ and $Q_{j}^{k}$. The low and high pass filters are denoted as $H$ and $G$. The single branch reconstructions reconstruct each packet atthe last decomposition level to the original level. The other packet values of similar levels are expressed to be zero if the construction is an inverse of the decomposition process.

\subsection{Long short-term memory networks:}

Enhancement of Recurrent Neural Network (RNN) is called long short-term memory (LSTM). Instead of conventional RNN units, the LSTM accomplishes memory blocks to solve exploding 
gradient and vanishing problems [15]. The network of LSTM memorizes and links the existing data to information received in the current stage [16]. Three gates such as input, forget and output gates are combined to form the LSTM. The current input is considered as $x_{j}$ the new and previous cell states are denoted as $y_{j}$ and $y_{j-1}$. The current and previous outputs are denoted as $h_{j}$ and $h_{j-1}$. Fig 1 explains the internal architecture of LSTM. The following equations explain the input gate principle of LSTM.

$$
\begin{gathered}
I_{j}=\delta\left(W_{j} \bullet\left[h_{j-1}, x_{j}\right]+a_{j}\right. \\
\tilde{y_{j}}=\tanh \left(W_{j} \bullet\left[h_{j-1}, x_{j}\right]+a_{j}\right. \\
y_{j}=\tanh \left(W_{j} \bullet\left[h_{j-1}, x_{j}\right]+a_{j}\right.
\end{gathered}
$$

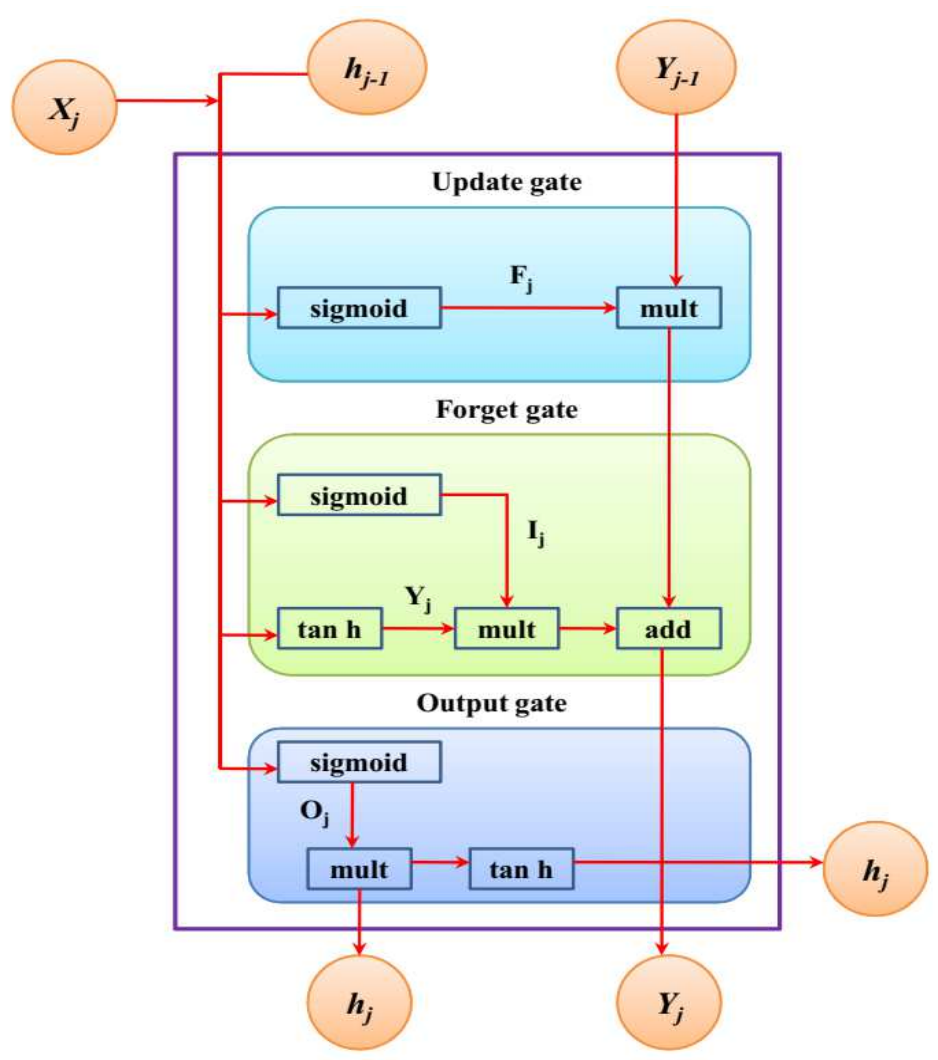

Fig 1: Basic architecture of LSTM

The sigmoid layer is used to pass $h_{j-1}$ and $x_{j}$. After $h_{j-1}$ and $x_{j}$ are passed via tanh layer, equation (5) is used to obtain the new information. Equation (6) combines $\tilde{y_{j}}$ long-term memory information $y_{j-1}$ into $y_{j}$. The input gate bias of LSTM and weight matrices are represented as $a_{j}$ and $W_{j}$. The dot product and sigmoid layer allow the selective passage of information with the help of the LSTM forget gate. Equation (7) executes the forget-based data from an existing cell 
with a certain probability. The sigmoid function, offset and weight matrix are denoted as $\delta, a_{j}$ and $W_{j}$.

$$
F_{j}=\sigma\left(W_{j} \bullet\left[h_{j-1}, y_{j}\right]+a_{j}\right.
$$

According to equations (8) and (9), the inputs $h_{j-1}$ are $y_{j}$ determined by the LSTM output gates. The tanh layer passes new information $y_{j}$.

$$
\begin{gathered}
O_{j}=\sigma\left(W_{o} \bullet\left[h_{j-1}, y_{j}\right]+a_{o}\right. \\
F_{j}=O_{j} \tanh \left(y_{j}\right)
\end{gathered}
$$

The LSTM, output gates weighted matrices are denoted as $W_{o}$ and $a_{o}$.

\subsection{Archimedes optimization algorithm (AOA) for multiple single LSTM networks:}

In the first step, the original image is decomposed by applying WPD and various reconstruction models are taken up. The performance of bean disease classification is improved by incorporating the combined multiple single LSTM network strength [21]. The classification results are obtained from every LSTM network after inputting the decomposed images. The final classification results are obtained by summing this LSTM network. Instead of summing, apply the Archimedes optimization algorithm (AOA) to enhance the classification accuracy. The AOA is used to evaluating the weight coefficient of each model. The mean absolute percentage error (MAPE) minimization determines the optimized weight coefficient. The image series minimizing standards and the error values determines it [22]. The MAPE minimization calculates a weight coefficient of the combined classification model. TheArchimedes optimization algorithm is mainly based on the Archimedes principle which is an interesting law of physics. The immersed objects are considered as a population of individuals in AOA. The accelerations, densities, random volumes with the initial population of objects are commenced in AOA [17]. Both exploitation and exploration processes are encompassed in AOA, which is considered a global optimization algorithm. The AOA updates the volume and density of every object in different iterations. The mathematical formulation of AOA stages are delineated as follows:

\subsubsection{Initialization:}

Equation (1) is used to initializing the position of each object.

$$
O B_{j}=L_{j}+\operatorname{random} \times\left(U_{j}-L_{j}\right) ; j=1,2, \ldots, M
$$

The $j^{\text {th }}$ population of $\mathrm{M}$ objects is $O B_{j}$. The upper and lower limits of a search space are denoted as $U_{j}$ and $L_{j}$. Equation (11) and (12) is used to update the density (D) and volume (V). 


$$
\begin{gathered}
D_{j}=\text { random } \\
V_{j}=\text { random }
\end{gathered}
$$

The D-dimensional vector creates the random interval as 0 to 1 and is denoted as random. Equation (13) updates the acceleration (A) of $j^{\text {th }}$ objects [14].

$$
A_{j}=L_{j}+\text { random } \times\left(U_{j}-L_{j}\right)
$$

The best fitness value with the objects is selected there also an initial population is evaluated. Assign $A_{\text {best }}, V_{\text {best }}, D_{\text {best }}$ and $y_{\text {best }}$. The fitness function of this work is optimal accuracy.

$$
\text { Fitness }=1-\text { Accuracy }
$$

\subsubsection{Density and volume update:}

Equation (15) is used to update the volume and density of $j^{\text {th }}$ objects for the $t+l$ iteration [18].

$$
\begin{gathered}
D_{j}^{t+1}=D_{j}^{t}+\text { random } \times\left(D_{\text {best }}-D_{j}^{t}\right) \\
V_{j}^{t+1}=V_{j}^{t}+\text { random } \times\left(V_{\text {best }}-V_{j}^{t}\right)
\end{gathered}
$$

Here, the best volume and density are $V_{j}^{t+1}$ and $D_{j}^{t+1}$ connected to the best objects.

\subsubsection{Density factor and transfer operator:}

The collision between objects occurs at first and the objects try to reach an equilibrium state after some time. The transfer operator $T f$ with AOA implements this. Equation (17) transforms search space from exploration to exploitation.

$$
T f=\exp \left(t-t_{\max } / t_{\max }\right)
$$

Here, the number of iteration and the maximum number of iteration are denoted as $t$ and $t_{\max }$. Where $D d$ is denoted as the density decreasing factor.

$$
D d^{t+1}=\exp \left(t-t_{\max } / t_{\max }\right)-\left(t / t_{\max }\right)
$$

\subsubsection{Exploration stage:}

The collision between objects occurs if $T f \leq 0.5$, then selects the random material $R M$. Equation (19) updates theacceleration of the object for the $t+l$ iteration. 


$$
A^{t+1}=\frac{D_{R m}+V_{R m} \times A_{R m}}{D_{j}^{j+1} \times V_{j}^{j+1}}
$$

Hence, the volume, density, and acceleration of random material are expressed as $V_{R m}$, $D_{R m}$ and $A_{R m}$. During one-third of iterations, the Tfguarantees an exploration.

\subsubsection{Exploitation stage:}

There is no collision between the objects if $T f>0.5$. Equation (20) updates the acceleration of the object for the $\mathrm{t}+1$ iteration.

$$
A_{j}{ }^{t+1}=\frac{D_{\text {best }}+V_{\text {best }} \times A_{\text {best }}}{D_{j}^{j+1} \times V_{j}^{j+1}}
$$

Here, the best object acceleration is $A_{\text {best }}$.

\subsubsection{Acceleration normalizing:}

Equation (21) calculates the percentage of normalizing acceleration.

$$
A_{j \text {-acceleration }}^{t+1}=\alpha \times \frac{A_{j}^{t+1}-\min (A)}{\max (A)-\min (A)}+\beta
$$

Where the normalization range is denoted as $\alpha$ and $\beta$.

\subsubsection{Updating position:}

Equation (22) updates the position of the object if $T f \leq 0.5$ (exploration stage).

$$
y_{j}^{j+1}=y_{j}^{t}+D_{2} \times \text { random } \times A_{j-\text { normalizaton }}^{t+1} \times D d \times\left(y_{\text {random }}-y_{j}^{t}\right)
$$

The constant term is denoted as $D$. Equation (23) updates the position of the object if Tf $>0.5$ (exploitation stage).

$$
y_{j}^{j+1}=y_{\text {best }}^{t}+E \times D_{2} \times \text { random } \times A_{j-\text { normalizaton }}^{t+1} \times D d \times\left(T \times y_{\text {random }}-y_{j}^{t}\right)
$$

Equation (23) is used to change the flag $\mathrm{F}$ to the direction of motion.

$$
F=\left\{\begin{array}{lll}
1 & \text { if } & P \leq 0.5 \\
-1 & \text { if } & P>0.5
\end{array}\right.
$$

Where, $P=2 \times$ random $-D_{4}$.

\subsubsection{Validation:}


The objective function evaluates each object. Assign $A_{\text {best }}, V_{\text {best }}, D_{\text {best }}$ and $y_{\text {best }}$. The steps involved in AOA for multiple single LSTM networks are depicted in algorithm 1.

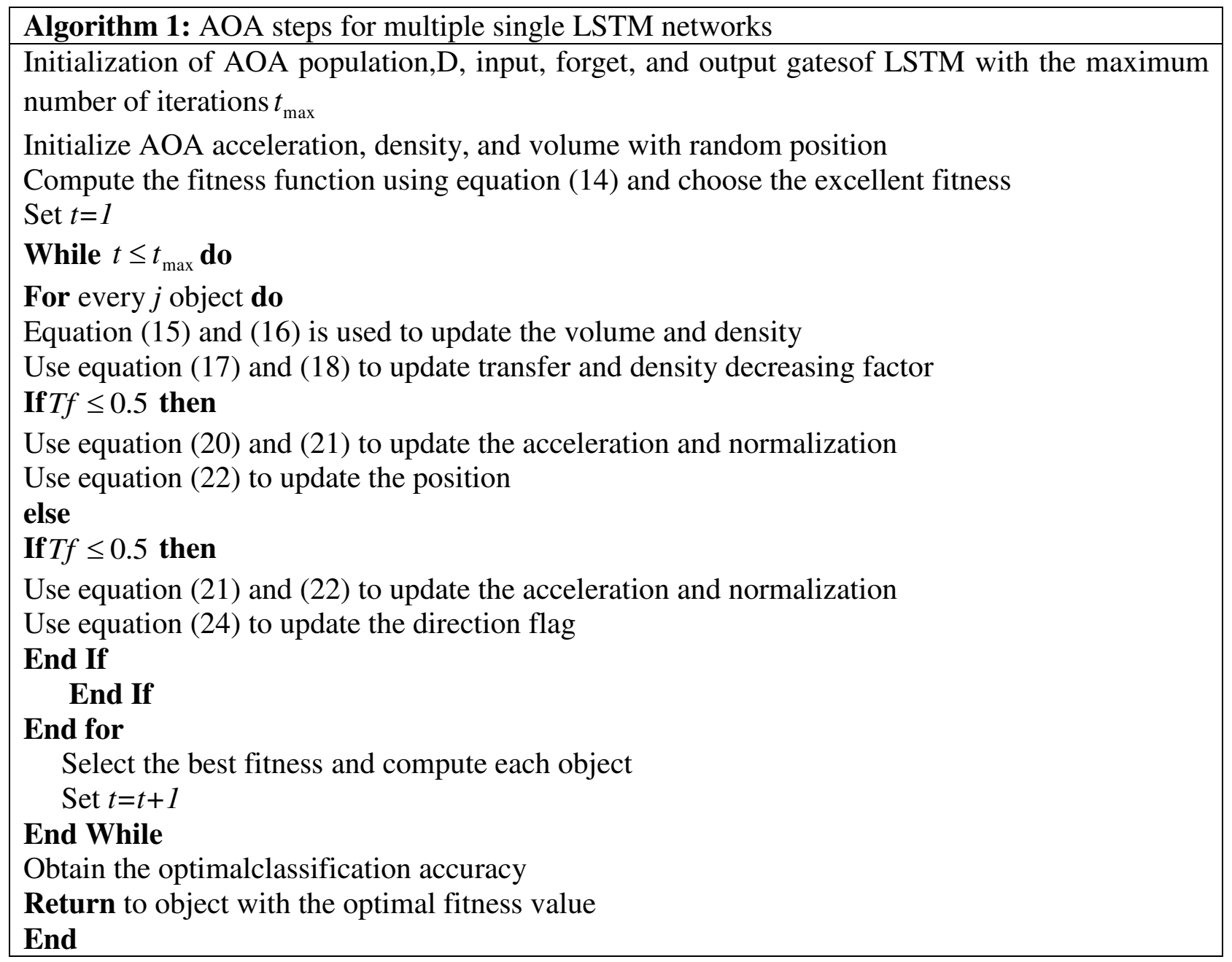

\subsection{Hybrid deep learning model (WPD-LSTM) for classification model:}

The WPD is used to decompose the image series in the proposed model. Mother wavelet utilizes a frequency-based third-order Daubechies type wavelet function (db3). In the classification image series, different frequency bands regularly exist simultaneously. The classification image series are separated in to high and low-frequency components after decomposition through WPD [23]. Some random output and fast varying image output are represented using high-frequency components and the low-frequency components denote the image output trend. The historical image output is an input model used in the proposed hybrid learning model [24, 25]. the relationship among bean image disease classification will be considered in this way. Fig 2 explains the hybrid deep learning with the AOA model for bean disease classification.

(i) The original image is decomposed using WPD. The original image output series is denoted as $Y=\left(I_{j-M+1},+I_{j-M+2}, \ldots ., I_{j}\right)^{T}$. Here, the bean image at time $t$ is $I_{j}$ and time 
horizon is $M$. The decomposed into a range of series with WPD is $Y$. The image output time series is decomposed into four series to consider the decomposition effect and computational efficiency. The WPD with $y_{j}$ decomposed series is rebuilt into four series. Where, $R_{j}=\left(I_{j, t-M+1}, I_{j, t-M+2}, \ldots ., I_{j, t}\right)^{T}$ describes a sub-series $j$.

(ii) From step (i), develop an independent LSM network for each sub-series. The inputs of each LSTM network consider the image data $R_{j}=\left(I_{j, t-M+1}, I_{j, t-M+2}, \ldots, I_{j, t}\right)^{T}$. Where, $I V_{j}=\left(I_{j}, b_{1}, b_{2, \ldots}, b_{k}\right)$ are the input variables of each LSTM. The deep learning model adopts four independent well trained three-layer LSTM networks. The classification result provided these independent LSTM networks.

(iii) A final classification result of bean disease classification is acquired by adopting an Archimedes optimization algorithm (AOA).

(iv) Table 2: Parameter settings for HDL-AOA

\begin{tabular}{|c|c|}
\hline Parameters & Ranges \\
\hline Type of transformation function & Mother wavelet \\
\hline Decomposition level & 4 \\
\hline LSTM batch size & 60 \\
\hline Halving factor & 0.9 \\
\hline Momentum & 0.8 \\
\hline Learning rate & 0.00002 \\
\hline Objective number & 30 \\
\hline $\mathrm{D}_{1}$ & 2 \\
\hline $\mathrm{D}_{2}$ & 6 \\
\hline $\mathrm{D}_{3}$ & 2 \\
\hline $\mathrm{D}_{4}$ & 0.5 \\
\hline Number of iteration & Maximum \\
\hline
\end{tabular}

\section{Experimental Analysis:}

In this work, we have proposed hybrid deep learning with the AOA (HDL-AOA) model for bean disease classification in which the proposed model performances are evaluated using various types of performance evaluation metrics and comparative analysis. The tensorFlow2 on an Intel(R) Core(TM) $17-2.2 \mathrm{GHz}$ processor with Python and Keras package implement the proposed HDL-AOA for bean disease classification. Additionally, the 4G and 16 GB RAM with graphical processing unit (GPU) NVIDIA GTX 1050 execute the experiments. [26] The experimental investigation in terms of classification performance analysis and comparative 
analysis are delineated in the following section. Table 2 delineates the parameter used for bean disease classification.

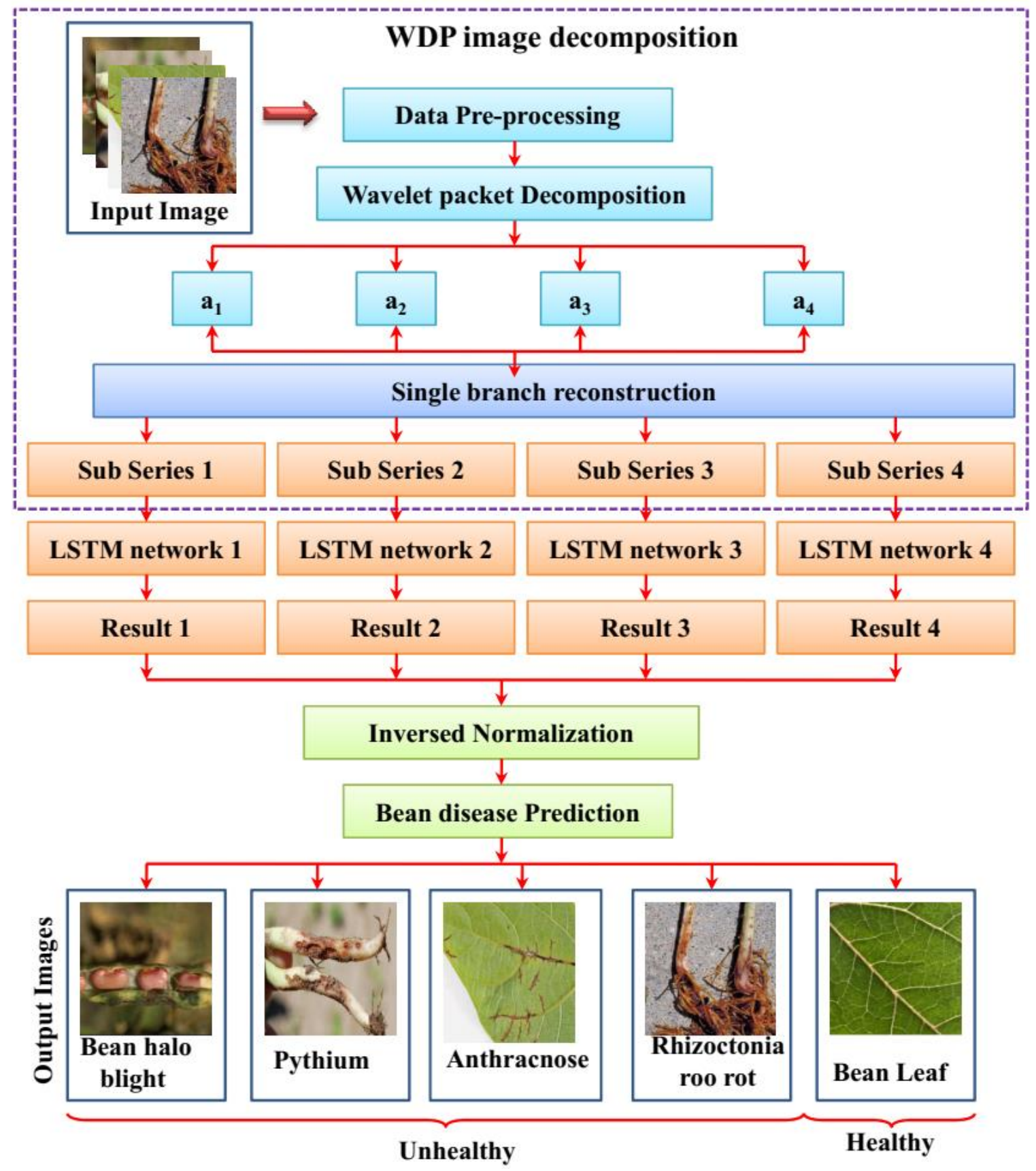

Fig 2: Proposed hybrid deep learning with AOA model for bean disease classification

\subsection{Dataset explanation:}

In this work, the dataset images were taken from Soybean (Large) Data Set [19] and [20] (https://www.ag.ndsu.edu/publications/crops/dry-edible-bean-disease-diagnostic-series\#section12). This dataset consists of 19 classes of attributes. From this, we have selected a single healthy 
class with four unhealthy classes such as Bean halo blight, Pythium diseases, Rhizoctonia root rot, and Anthracnose (Fig 3). The training and testing image details based on the dataset are delineated in Table 3. Table 4 explains the bean disease symptoms.

Table 3: Dataset description

\begin{tabular}{|c|c|c|c|}
\hline Name of the class & Training set & Testing set & Total set \\
\hline Healthy & 820 & 230 & 1050 \\
\hline Bean halo blight & 670 & 150 & 820 \\
\hline Pythium diseases & 690 & 200 & 890 \\
\hline Rhizoctonia root rot & 1200 & 250 & 1450 \\
\hline Anthracnose & 795 & 300 & 1095 \\
\hline
\end{tabular}

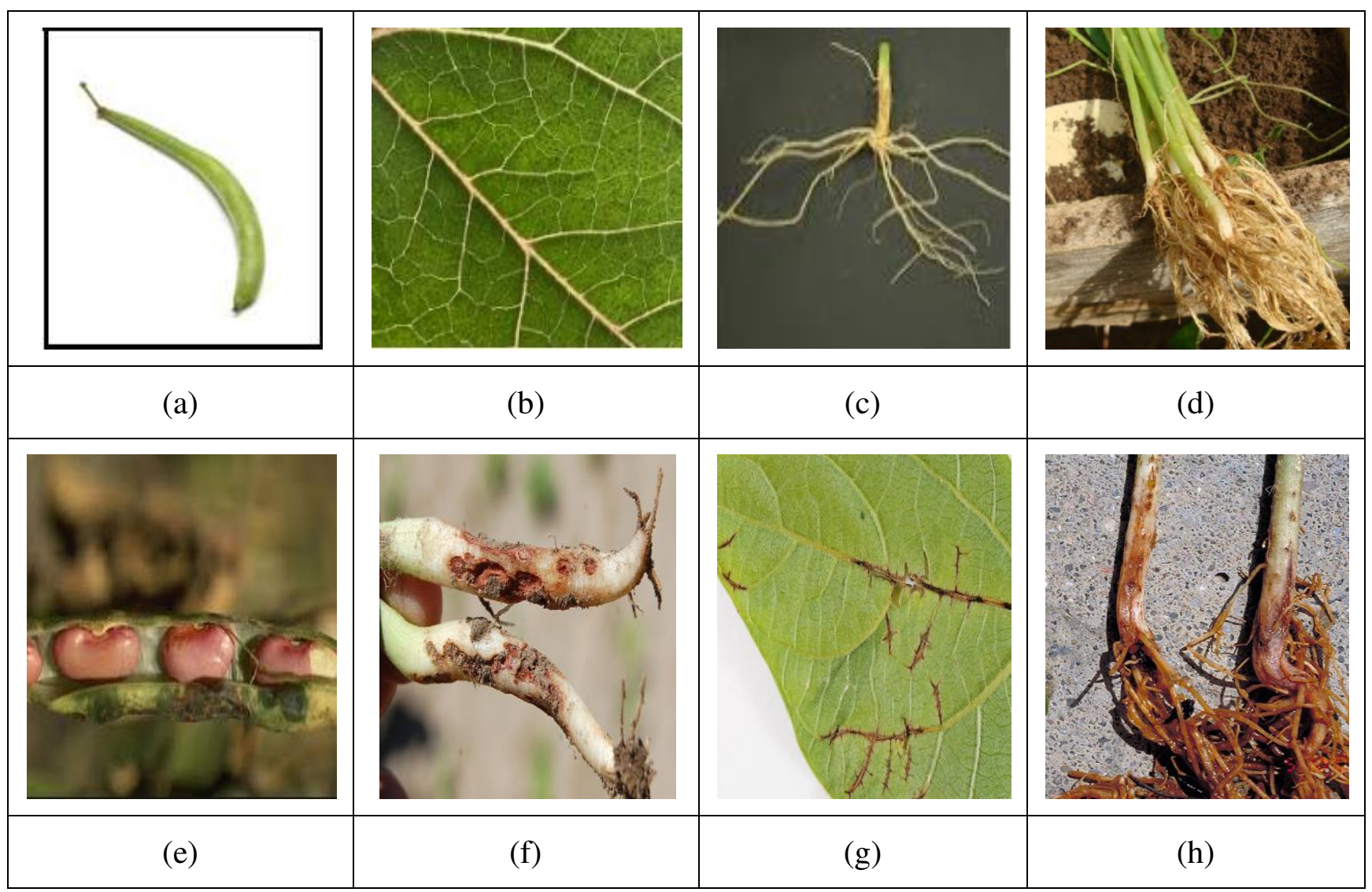

Fig 3: Sample data description, (a) Healthybean and (e) Bean halo blight, (b) Healthybean root and (f) Pythium disease affected root, (c) Healthy bean leaf and (g) Anthracnose disease affected bean leaf, (d) Healthy bean root and (h) Rhizoctonia root rot

\subsection{Evaluation measures:}


The performance efficiency of the proposed model is evaluated using different kinds of evaluation metrics such as accuracy, specificity, sensitivity, precision, recall, F-score, and MAPE. Each measure is delineated as follows:

$$
\text { Accuracy }=\frac{\text { True positive }+ \text { True negative }}{\text { True positive }+ \text { Truenegative }+ \text { False positive }+ \text { False negative }}
$$

The specificity, sensitivity, precision, and recall equations are delineated in equations (26) to (29).

$$
\begin{aligned}
\text { Specificity } & =\frac{\text { True positive }}{\text { True positive }+ \text { False negative }} \\
\text { Sensitivity } & =\frac{\text { True negative }}{\text { True negative }+ \text { False positive }} \\
\operatorname{Pr} \text { ecision } & =\frac{\text { True positive }}{\text { True positive }+ \text { False positive }} \\
\operatorname{Re} \text { call } & =\frac{\text { True positive }}{\text { True positive }+ \text { False negative }}
\end{aligned}
$$

Table 4: Disease symptoms

\begin{tabular}{|c|c|}
\hline Name of the diseases & Symptoms \\
\hline Bean halo blight & $\begin{array}{r}\text { Broad yellow-green halo may develop around necrotic spots, } \\
\text { which may infect the infect pods and seeds }\end{array}$ \\
\hline Pythium disease & $\begin{array}{r}\text { Water-soaked necrotic areas on roots or hypocotyls and initial } \\
\text { root rot symptoms appear as elongated }\end{array}$ \\
\hline Anthracnose & Dark and slender leaf vein and petiole lesions \\
\hline Rhizoctonia root rot & Internal brick-red discoloration of pith \\
\hline
\end{tabular}

Where the number of instances that belong to the correctly identified class is True Positive (TP). The number of instances that belong to the incorrectly identified class isFalse Negative (FN). The number of instances that do not tend to the class, which are incorrectly identified is called False Positive (FP). Similarly, the number of instances not tends to the class, which are correctly identified are called True Negative (TN). Equation (30) and (31) explains the F-score value and the mean absolute percentage error (MAPE).

$$
F-\text { score }=2 * \frac{P_{\text {recison }} * R_{\text {ecall }}}{P_{\text {recison }}+R_{\text {ecall }}}
$$




$$
M A P E=\frac{1}{M} \sum_{t=1}^{M} \frac{\left|R_{j}-\hat{R}_{j}\right|}{R_{j}} \times 100 \%
$$

Where, the true and forecasted values are defined as $R_{j}$ and $\hat{R}_{j}$. The classification horizon is $M$ at time $t$.

\subsection{Performance Analysis:}

The performance analysis with respect to accuracy and loss is depicted in Fig 4. According to the ratio of 70/30 divides both training and testing set. The input images were resized into $224 \times 224$ pixels. The training and testing performance in terms of accuracy and loss are depicted in Fig 4 (a) and (b). There are 30 epochs chosen for this experiment. Fig 4 (a) takes 30 epochs with 100\% accuracy in which the accuracy levels varying from $0.65 \%$ to $0.98 \%$ training accuracy and the testing accuracy varies from $0.83 \%$ to $0.92 \%$. According to Fig 4 (b), the training loss varies from $0.82 \%$ to $1.0 \%$. Similarly, the testing loss varies from $0.45 \%$ to $0.23 \%$. The proposed HDLAOA takes better training accuracy and testing accuracy as well as training loss and testing loss in terms of bean disease classification.

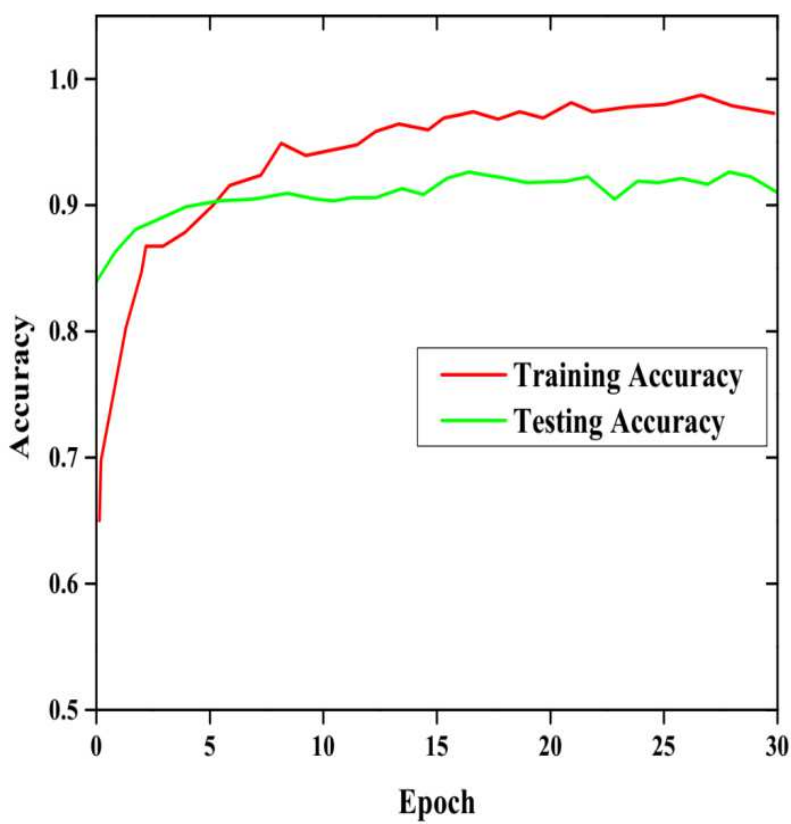

(a)

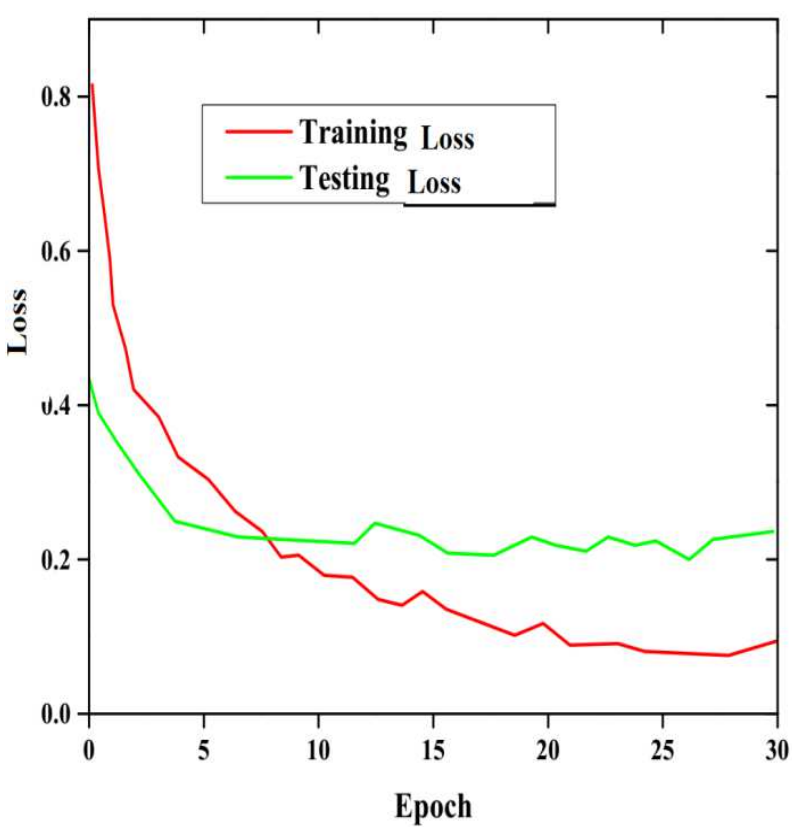

(b)

Fig 4: Performance analysis, (a) Accuracy and (b) Loss

Fig 5 depicts the proposed HDL-AOA performance analysis with respect to each class. We have selected a healthy bean plant with four unhealthy images belongs to Bean halo blight, Pythium diseases, Rhizoctonia root rot, Anthracnose diseases. The accuracy, specificity, and 
sensitivity performance of each class are evaluated. For a healthy class, we have obtained $84 \%$ accuracy, $89 \%$ specificity, and $91 \%$ sensitivity results. We have obtained $89 \%$ accuracy, $81 \%$ specificity, and $90 \%$ sensitivity results for the bean halo blight class. The Pythium class provided $91 \%$ accuracy, $84 \%$ specificity, and $81 \%$ sensitivity outcomes. For Rhizoctonia root rot, we have attained $91 \%$ accuracy, 95\% specificity, and $84 \%$ sensitivity results. Similarly, 92\% accuracy, $88 \%$ specificity, and $95 \%$ sensitivity results are obtained for Anthracnose. The Confusion matrix with respect to each class is delineated in Fig 6.

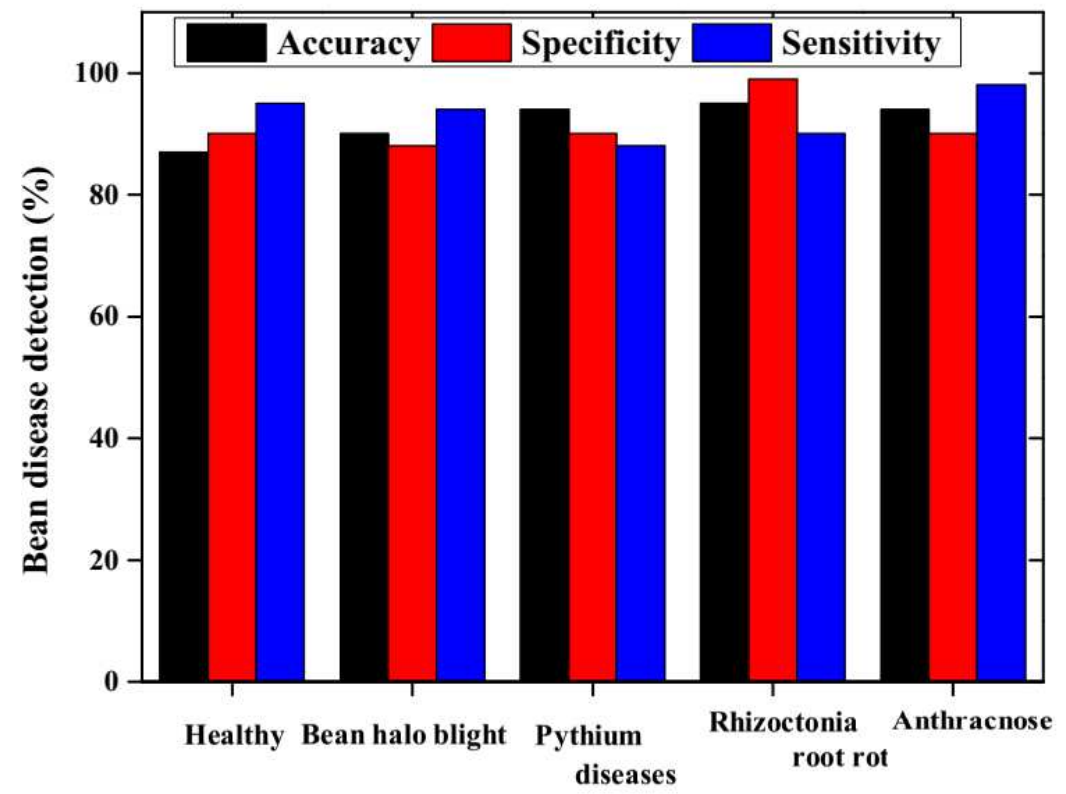

Fig 5: Proposed HDL-AOA performance analysis with respect to each class

\begin{tabular}{r|c|c|c|c|c|}
\cline { 2 - 5 } & Healthy & $\begin{array}{c}\text { Bean halo } \\
\text { blight }\end{array}$ & Pythium & $\begin{array}{c}\text { Rhizoctonia } \\
\text { root rot }\end{array}$ & Anthracnose \\
\cline { 2 - 6 } $\begin{array}{r}\text { Healthy } \\
\text { Bean halo blight }\end{array}$ & $84 \%$ & $4.5 \%$ & $8.12 \%$ & $2.12 \%$ & $1.26 \%$ \\
\cline { 2 - 6 } $\begin{array}{r}\text { Pythium diseases } \\
\text { Rhizoctonia root rot } \\
\text { Anthracnose }\end{array}$ & $4.15 \%$ & $1.66 \%$ & $91 \%$ & $3.19 \%$ & $0 \%$ \\
\cline { 2 - 6 } & $4.65 \%$ & $0 \%$ & $3.89 \%$ & $91 \%$ & $0.46 \%$ \\
\cline { 2 - 6 } & $3.78 \%$ & $1.12 \%$ & $3.01 \%$ & $0 \%$ & $92 \%$ \\
\hline
\end{tabular}

Fig 6: Confusion matrix with respect to each class

Fig 7 illustrates the proposed HDL-AOA performance analysis with respect to ROC. When analyzing the overall performance, the ROC curves plotted among true positive rate (TPR) and false-positive rate (FPR) respectively. Each class varies from 0.1 to 1.0. The area under ROC (AUC) is computed to be $9.9 \%$ for HDL-AOA models. Based on the ROC curve, we have obtained better true positive rates in terms of the healthy class, Bean halo blight, Pythium disease class, Rhizoctonia root rot class, Anthracnose class. 


\subsection{Comparative Analysis:}

Performance evaluation of MAPE with respect to various methods and each class are depicted in Fig 8. Different methods such as long short-term memory (LSTM), Recurrent neural network (RNN), Multiple layer perceptron (MLP), Convolutional neural network (CNN), and proposed HDL-AOA model were chosen to validate the performance of MAPE. The MAPE value of the proposed HDL-AOA model is averagely minimized compared with other methods such as LSTM, MLP, CNN, and RNN. Based on Fig 8, the proposed HDL-AOA achieves 2\% MAPE for healthy class, $2.1 \%$ for bean halo blight, $3 \%$ for Pythium, 5\% for Rhizoctonia, and $2 \%$ for Anthracnose. However, the proposed HDL-AOA model accomplishes low errors during bean disease classification.

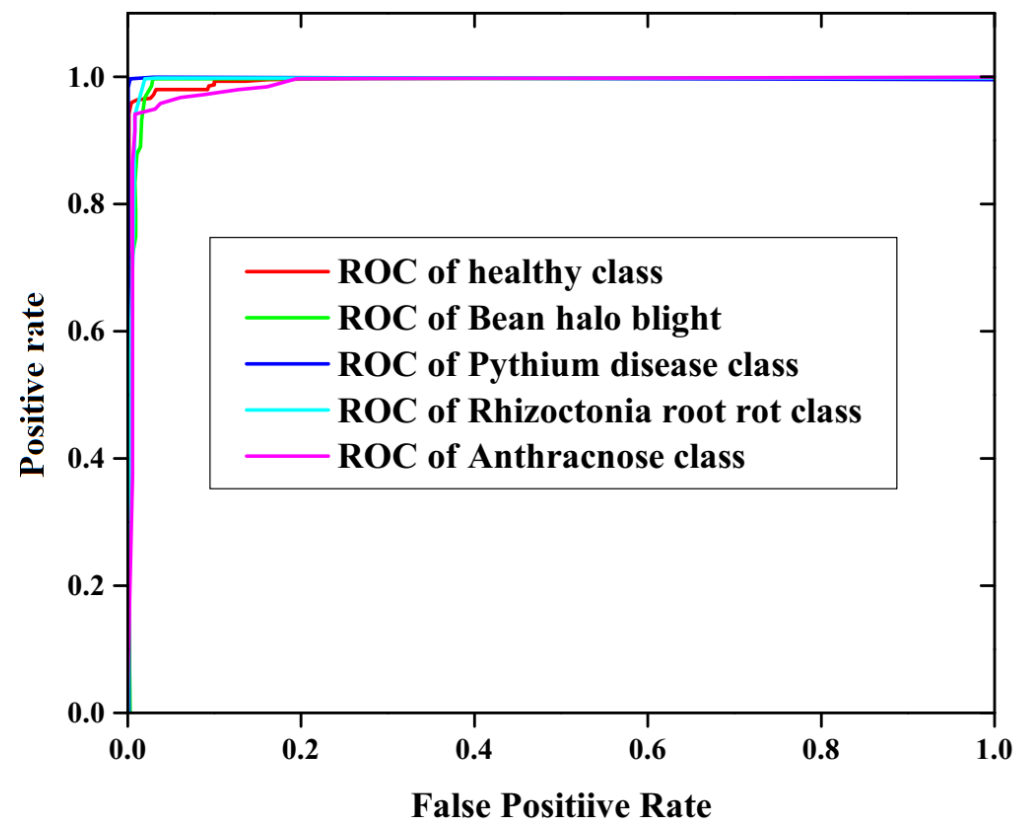

Fig 7: Proposed HDL-AOA performance analysis with respect to ROC

Table 5: State-of-comparison of classification

\begin{tabular}{|c|c|c|c|c|c|}
\hline \multirow{2}{*}{$\begin{array}{c}\text { Evaluation } \\
\text { measures }\end{array}$} & \multicolumn{5}{|c|}{ Name of the methods } \\
\cline { 2 - 6 } & RNN & DCNN & CNN & LSTM & HDL-AOA \\
\hline Accuracy & $89.01 \%$ & $98.01 \%$ & $96.89 \%$ & $97.13 \%$ & $98.23 \%$ \\
\hline Specificity & $76.89 \%$ & $95.12 \%$ & $93.14 \%$ & $95.09 \%$ & $97.13 \%$ \\
\hline Sensitivity & $91.89 \%$ & $93.12 \%$ & $89.92 \%$ & $97.92 \%$ & $98.01 \%$ \\
\hline Precision & $81.89 \%$ & $82.56 \%$ & $94.78 \%$ & $97.82 \%$ & $98.45 \%$ \\
\hline Recall & $92.45 \%$ & $97.23 \%$ & $82.66 \%$ & $98.41 \%$ & $99.12 \%$ \\
\hline F-score & $78.85 \%$ & $98.67 \%$ & $98.25 \%$ & $98.14 \%$ & $99.45 \%$ \\
\hline
\end{tabular}

Table 5 illustrates the state-of-art comparison of bean disease classification. In this experiment, we have chosen five state-of-art methods including Recurrent Neural Network 
(RNN) [27], Deep Convolutional Neural Network (DCNN) [7], Convolutional Neural Network (CNN) [9], Long Short Term Memory (LSTM) [26] and proposed HDL-AOA. The proposed HDL-AOA demonstrates $98.23 \%$ accuracy, $97.13 \%$ specificity, $98.01 \%$ sensitivity, $98.45 \%$ precision, $99.12 \%$ recall, and $99.45 \% \mathrm{~F}$-score values than other existing techniques.

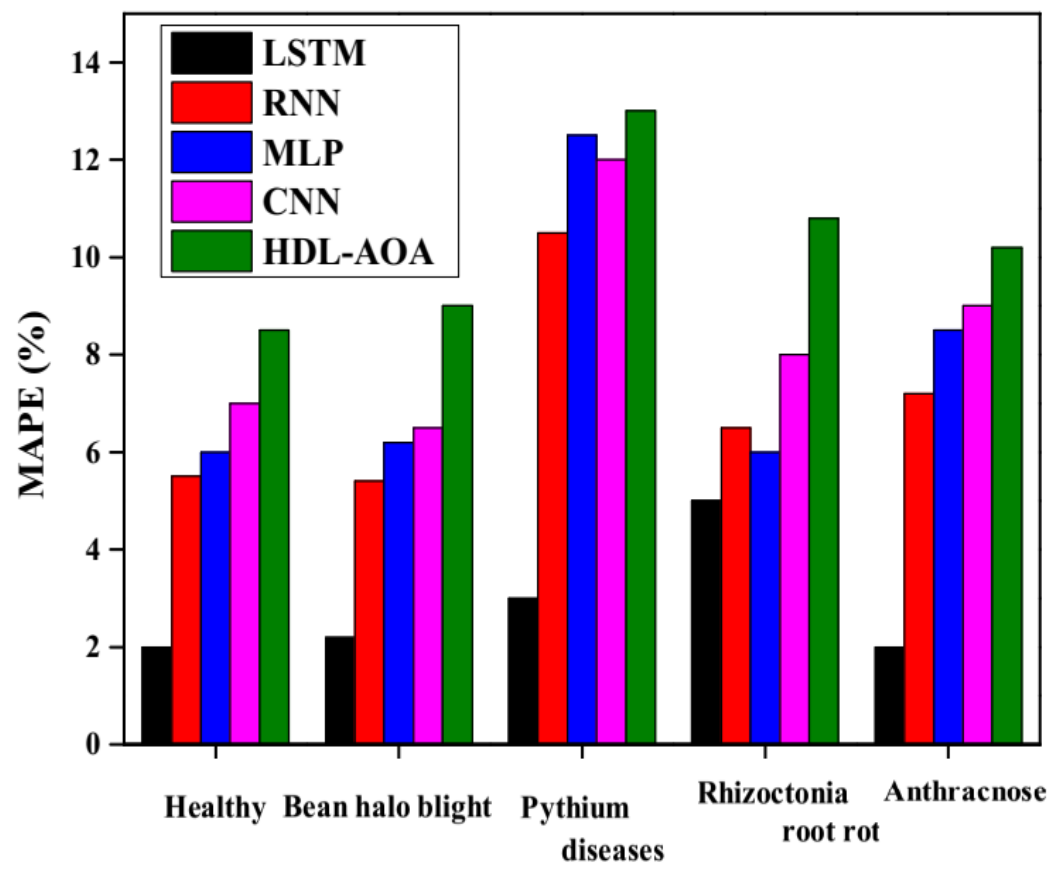

Fig 8: Performance evaluation of MAPE with respect to various methods and each class

\section{Conclusion:}

This paper proposed a hybrid deep learning model with Archimedes optimization algorithm (HDL-AOA) for bean disease classification.The four unhealthy image classes(Bean halo blight, Pythium diseases, Rhizoctonia root rot, and Anthracnose)along with the healthy class images were collected from Soybean (Large) Data Set. The HDL-AOA model for bean disease classification is implemented in MATLAB software. The proposed HDL-AOA achieves the MAPE values such as $2 \%$ for healthy class, $2.1 \%$ for bean halo blight, $3 \%$ for Pythium, $5 \%$ for Rhizoctonia, and $2 \%$ for Anthracnose than other existing methods such as LSTM, MLP, CNN, and RNN.The proposed HDL-AOA demonstrates $98.23 \%$ accuracy, $97.13 \%$ specificity, $98.01 \%$ sensitivity, $98.45 \%$ precision, $99.12 \%$ recall, and $99.45 \%$ F-score values than other existing techniques including RNN, DCNN, LSTM, and CNN.

Funding: Not applicable

Conflicts of interest Statement: Not applicable 


\section{Compliance with Ethical Standards}

\section{Conflict of interest}

The authors declare that they have no conflict of interest.

\section{Human and Animal Rights}

This article does not contain any studies with human or animal subjects performed by any of the authors.

\section{Informed Consent}

Informed consent was obtained from all individual participants included in the study.

Consent to participate: Not applicable

Consent for publication: Not applicable

\section{Availability of data and material:}

Data sharing is not applicable to this article as no new data were created or analyzed in this study.

\section{Reference:}

1. Argüeso, David, ArtzaiPicon, Unai Irusta, Alfonso Medela, Miguel G. San-Emeterio, ArantzaBereciartua, and Aitor Alvarez-Gila. (2020). Few-Shot Learning approach for plant disease classification using images taken in the field. Computers and Electronics in Agriculture 175: 105542.

2. H.Tavakoli, P. Alirezazadeh, A. Hedayatipour, AH BanijamaliNasib, and N. Landwehr. (2021). Leaf image-based classification of some common bean cultivars using discriminative convolutional neural networks. Computers and Electronics in Agriculture 181: 105935.

3. Kantale, Pooja, and ShubhadaThakare. (2020). A Review on Pomegranate Disease Classification Using Machine Learning and Image Segmentation Techniques. In 2020 4th International Conference on Intelligent Computing and Control Systems (ICICCS), pp. 455-460. IEEE.

4. P. Sahu, A.Chug, A. P. Singh, D. Singh, , \& R. P. Singh. (2020). Implementation of CNNs for Crop Diseases Classification: A Comparison of Pre-trained Model and Training from Scratch. IJCSNS, 20 10, 206. 
5. Brandt, Tracy, Laura M. Sack, Dolores Arjona, Duanjun Tan, Hui Mei, Hong Cui, Hua Gao et al. (2020). Adapting ACMG/AMP sequence variant classification guidelines for single-gene copy number variants. Genetics in Medicine 22 2: 336-344.

6. Kiptoo, Gaudencia J., Miriam G. Kinyua, Lexa G. Matasyoh, and Oliver K. Kiplagat. (2020). Morphological traits associated with anthracnose (Colletotrichum lindemuthianum) resistance in selected common bean (Phaseolus vulgaris L.) genotypes. African Journal of Plant Science 14, no. 2: 45-56.

7. S. Uğuz, and N. Uysal. (2020). Classification of olive leaf diseases using deep convolutional neural networks. Neural Computing and Applications, pp.1-17.

8. Esgario, G.M. José, A. Renato, Krohling, and José A. Ventura. (2020). Deep learning for classification and severity estimation of coffee leaf biotic stress. Computers and Electronics in Agriculture 169: 105162.

9. Esgario, G.M. José, A. Renato, Krohling, and José A. Ventura. (2020). Deep learning for classification and severity estimation of coffee leaf biotic stress. Computers and Electronics in Agriculture 169: 105162.

10. Hernández, S., and Juan L. López. (2020). Uncertainty quantification for plant disease detection using Bayesian deep learning. Applied Soft Computing 96: 106597.

11. Chen, Junde, Jinxiu Chen, Defu Zhang, Yuandong Sun, and YaserAhangariNanehkaran. (2020). Using deep transfer learning for image-based plant disease identification. Computers and Electronics in Agriculture 173: 105393.

12. Li, Kaizhou, Jianhui Lin, Jinrong Liu, and Yandong Zhao. (2020). Using deep learning for Image-Based different degrees of ginkgo leaf disease classification. Information 11 2: 95.

13. Coifman, R. Ronald, Yves Meyer, Steven Quake, and M. Victor Wickerhauser. (1994). Signal processing and compression with wavelet packets. In Wavelets and their applications, pp. 363-379. Springer, Dordrecht.

14. C. Zhitong, C. Hongping, H.Guoguang, , \& E. Ritchie, (August). (2001). Rotor fault diagnosis of induction motor based on wavelet reconstruction. In ICEMS'2001. Proceedings of the Fifth International Conference on Electrical Machines and Systems (IEEE Cat. No. 01EX501) 1:374-377. IEEE.

15. S. Hochreiter. (1998) . The vanishing gradient problem during learning recurrent neural nets and problem solutions. International Journal of Uncertainty, Fuzziness and Knowledge-Based Systems, 6 (02), 107-116.

16. Zhang, Nian, and Pradeep K. Behera. (2012). Solar radiation prediction based on recurrent neural networks trained by Levenberg-Marquardt backpropagation learning algorithm. In 2012 IEEE PES Innovative Smart Grid Technologies (ISGT), pp. 1-7. IEEE,

17. Y. Li, H.Zhu, D.Wang, K. Wang, W. Kong, and X. Wu. (2021). Comprehensive optimization of distributed generation considering network reconstruction based on 
Archimedes optimization algorithm. In IOP Conference Series: Earth and Environmental Science Vol. 647 : 1 012031. IOP Publishing.

18. Hashim, Fatma A., Kashif Hussain, Essam H. Houssein, Mai S. Mabrouk, and Walid AlAtabany. (2020). Archimedes optimization algorithm: a new metaheuristic algorithm for solving optimization problems. Applied Intelligence: 1-21.

19. D. Dua, and C. Graff. (2019). UCI Machine Learning Repository [http://archive.ics.uci.edu/ml]. Irvine, CA: University of California, School of Information and Computer Science.

20. S. G. Markell, R. M.Harveson, \& J. Pasche. (2016). Dry Edible Bean Disease Diagnostic Series.

21. H. Tavakoli, P. Alirezazadeh, A. Hedayatipour, AH BanijamaliNasib, and N. Landwehr. (2021). Leaf image-based classification of some common bean cultivars using discriminative convolutional neural networks. Computers and Electronics in Agriculture 181: 105935.

22. P. Kantale, and S. Thakare, (2020). May. A Review on Pomegranate Disease Classification Using Machine Learning and Image Segmentation Techniques. In 2020 4th International Conference on Intelligent Computing and Control Systems (ICICCS) pp. 455-460. IEEE.

23. Sahu, Priyanka, Anuradha Chug, Amit Prakash Singh, Dinesh Singh, and Ravinder Pal Singh. (2020). Implementation of CNNs for Crop Diseases Classification: A Comparison of Pre-trained Model and Training from Scratch. IJCSNS 20 10: 206.

24. Brandt, Tracy, Laura M. Sack, Dolores Arjona, Duanjun Tan, Hui Mei, Hong Cui, Hua Gao et al. (2020). Adapting ACMG/AMP sequence variant classification guidelines for single-gene copy number variants. Genetics in Medicine 22, 2: 336-344.

25. Kiptoo, J. Gaudencia, Miriam G. Kinyua, Lexa G. Matasyoh, and Oliver K. Kiplagat. (2020). Morphological traits associated with anthracnose (Colletotrichum lindemuthianum) resistance in selected common bean (Phaseolus vulgaris L.) genotypes. African Journal of Plant Science 14, 2: 45-56.

26. Turkoglu, Muammer, DavutHanbay, and Abdulkadir Sengur. (2019). Multi-model LSTM-based convolutional neural networks for detection of apple diseases and pests. Journal of Ambient Intelligence and Humanized Computing: 1-11.

27. D. Jayakumar, A. Elakkiya, R. Rajmohan, and M. O. Ramkumar. (2020). Automatic Prediction and Classification of Diseases in Melons using Stacked RNN based Deep Learning Model. In 2020 International Conference on System, Computation, Automation and Networking (ICSCAN), pp. 1-5. IEEE.

28. C. Mitchell, Diane, Frank R. Lawrence, Terryl J. Hartman, and Julianne M. Curran. (2009). Consumption of dry beans, peas, and lentils could improve diet quality in the US population.Journal of the American dietetic association 109 5: 909-913.

29. Kabagambe, K. Edmond, Ana Baylin, Edward Ruiz-Narvarez, XiniaSiles, and Hannia Campos. (2005) . Decreased consumption of dried mature beans is positively associated 
with urbanization and nonfatal acute myocardial infarction. The Journal of nutrition 135 7: 1770-1775.

30. G. Grant, P. M. Dorward, W. C. Buchan, J. C. Armour, \& A. Pusztai. (1995) . Consumption of diets containing raw soya beans (Glycine max), kidney beans (Phaseolus vulgaris), cowpeas (Vigna unguiculata) or lupin seeds (Lupinus angustifolius) by rats for up to 700 days: effect on body composition and organ weights. British Journal of Nutrition, 73 (1), 17-29.

31. Lindgren, B. PETER, RICHARD C. Peet, and NICKOLAS J. (1986). Panopoulos. Gene cluster of Pseudomonas syringaepv. phaseolicola controls pathogenicity of bean plants and hypersensitivity of nonhost plants. Journal of Bacteriology 168 2: 512-522. 
Figures

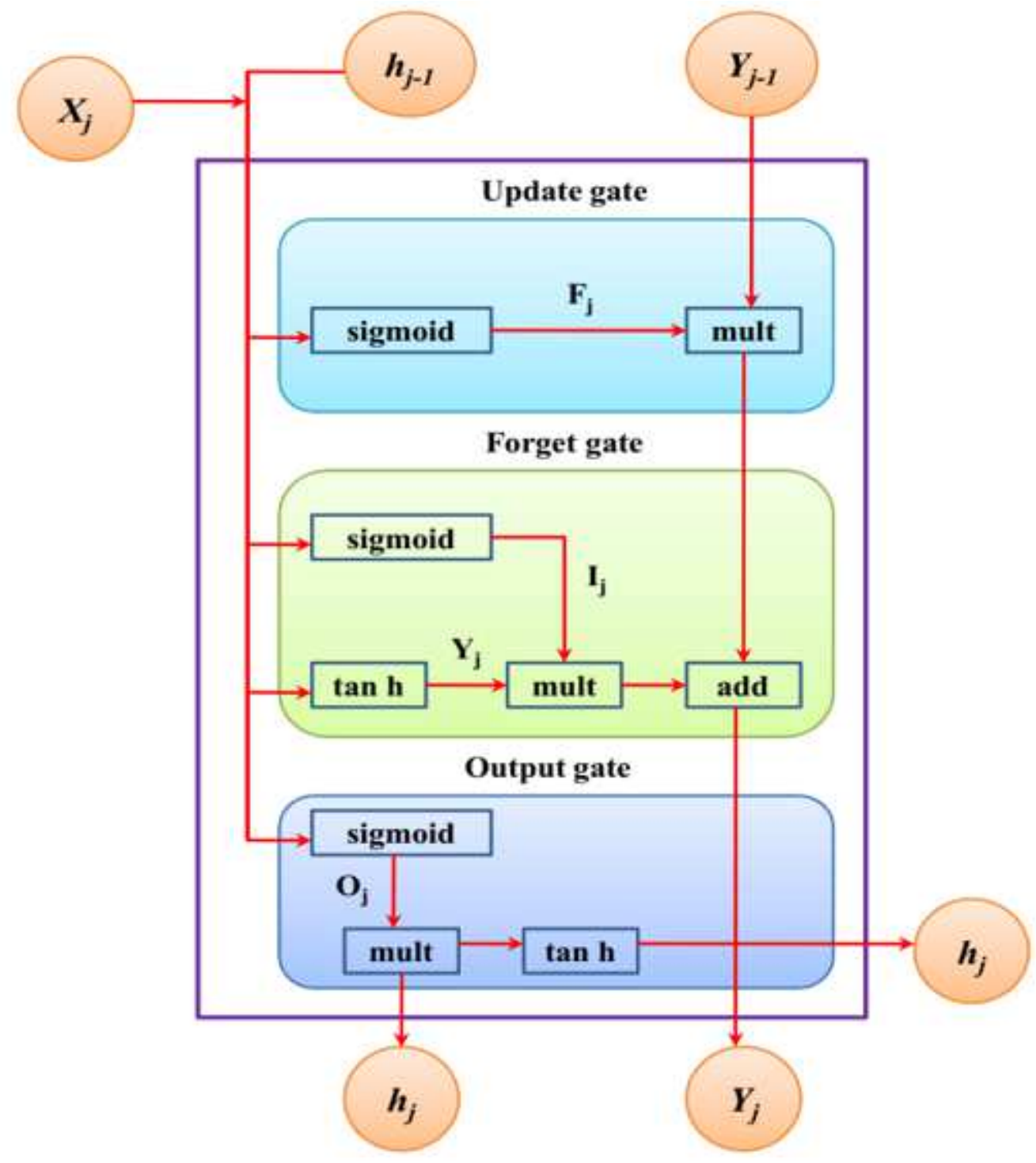

Figure 1

Basic architecture of LSTM 


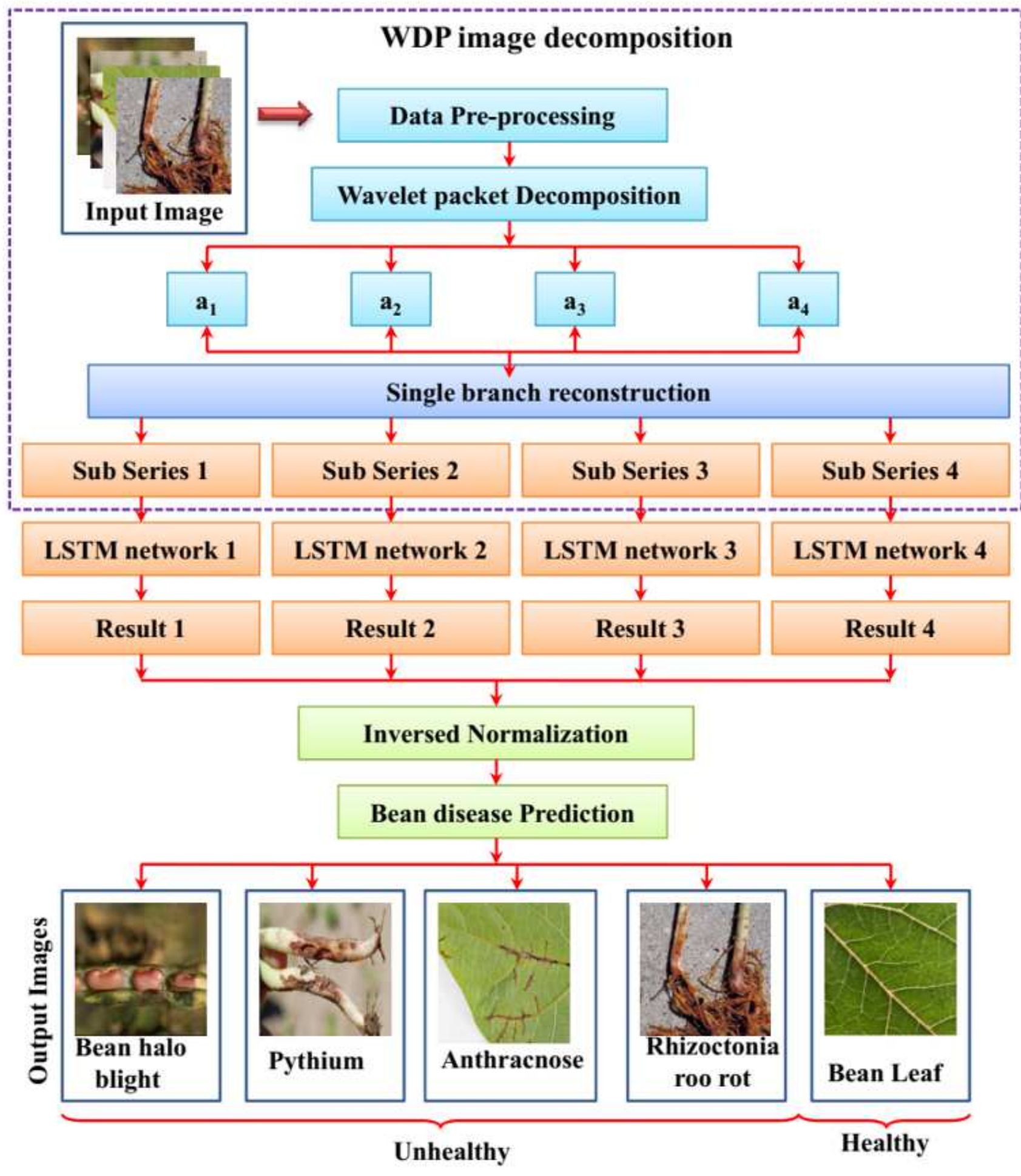

Figure 2

Proposed hybrid deep learning with AOA model for bean disease classification 


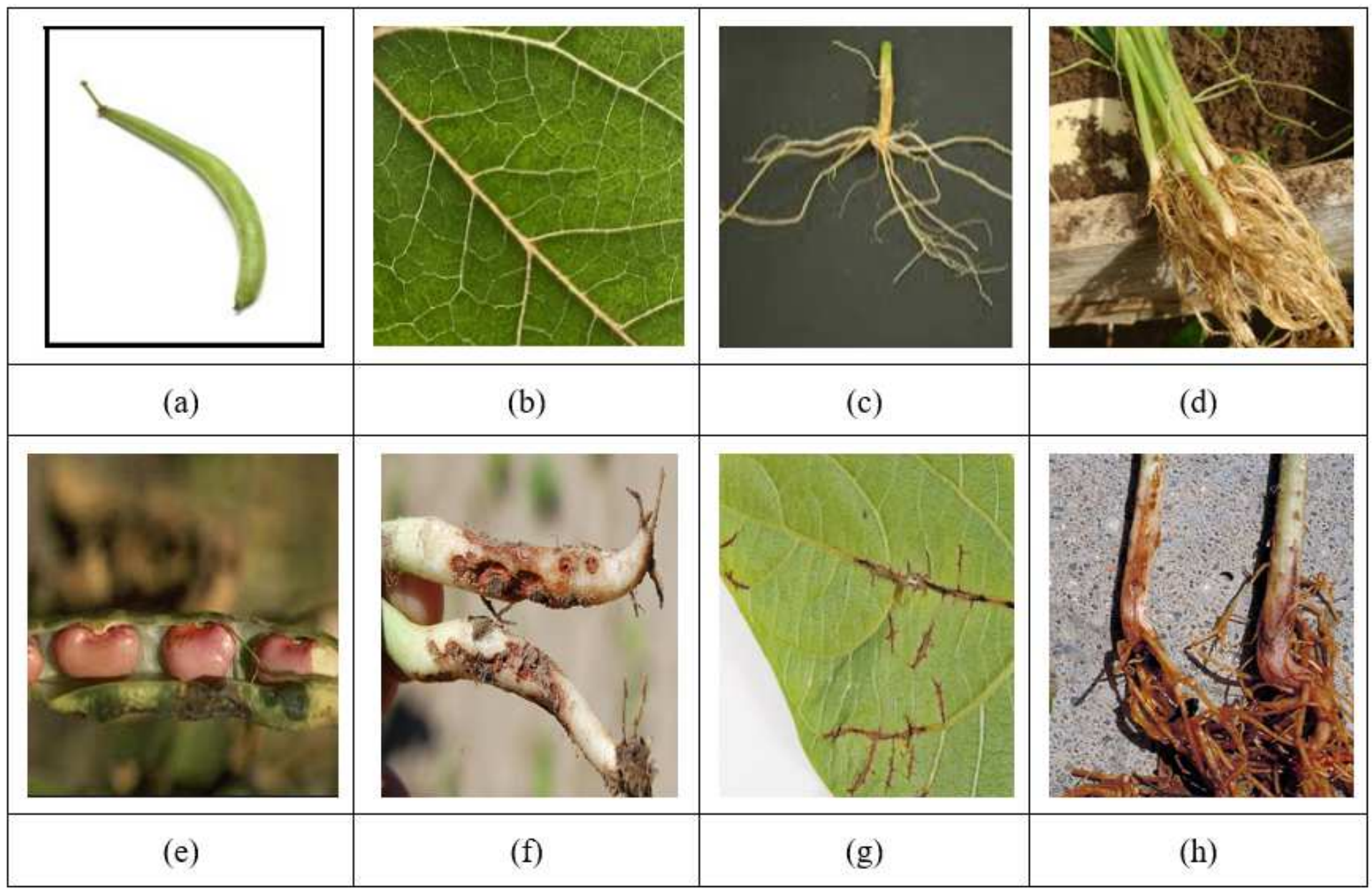

Figure 3

Sample data description, (a) Healthybean and (e) Bean halo blight, (b) Healthybean root and (f) Pythium disease affected root, (c) Healthy bean leaf and (g) Anthracnose disease affected bean leaf, (d) Healthy bean root and $(\mathrm{h})$ Rhizoctonia root rot 


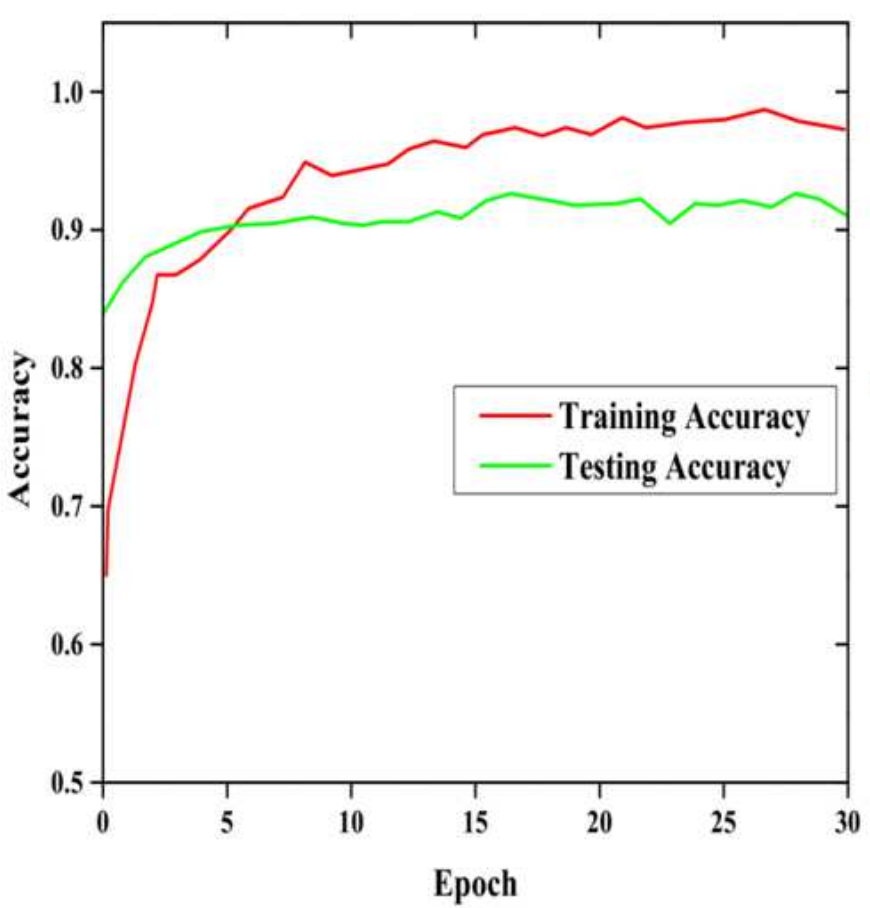

(a)

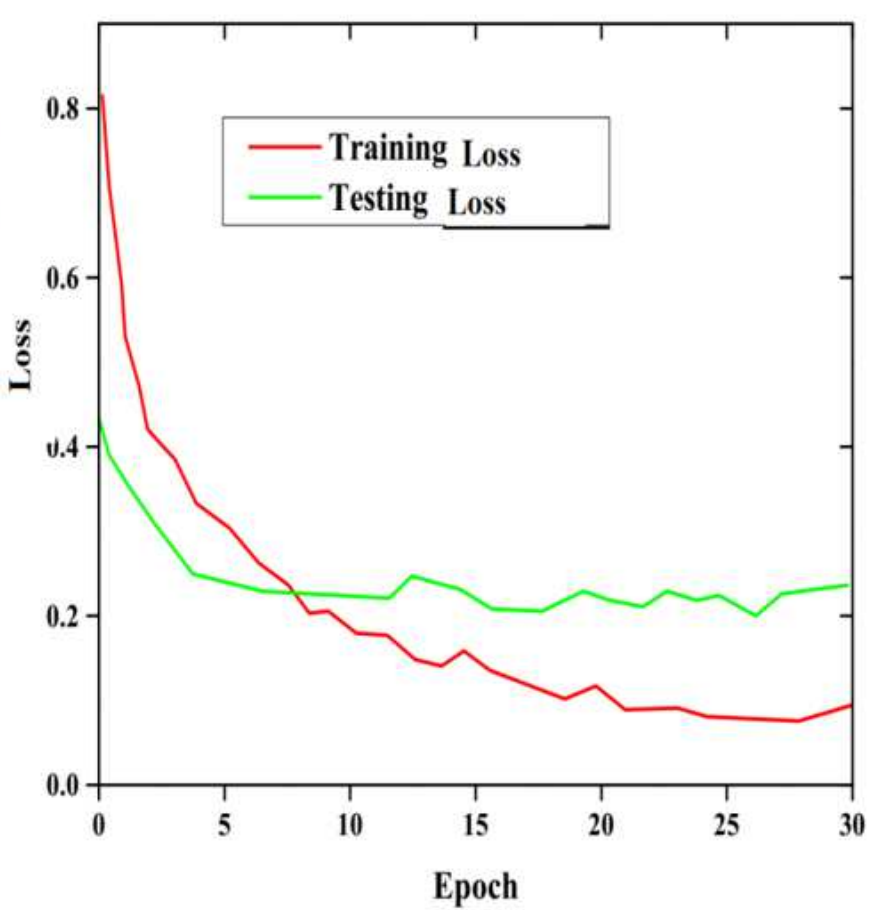

(b)

\section{Figure 4}

Performance analysis, (a) Accuracy and (b) Loss 


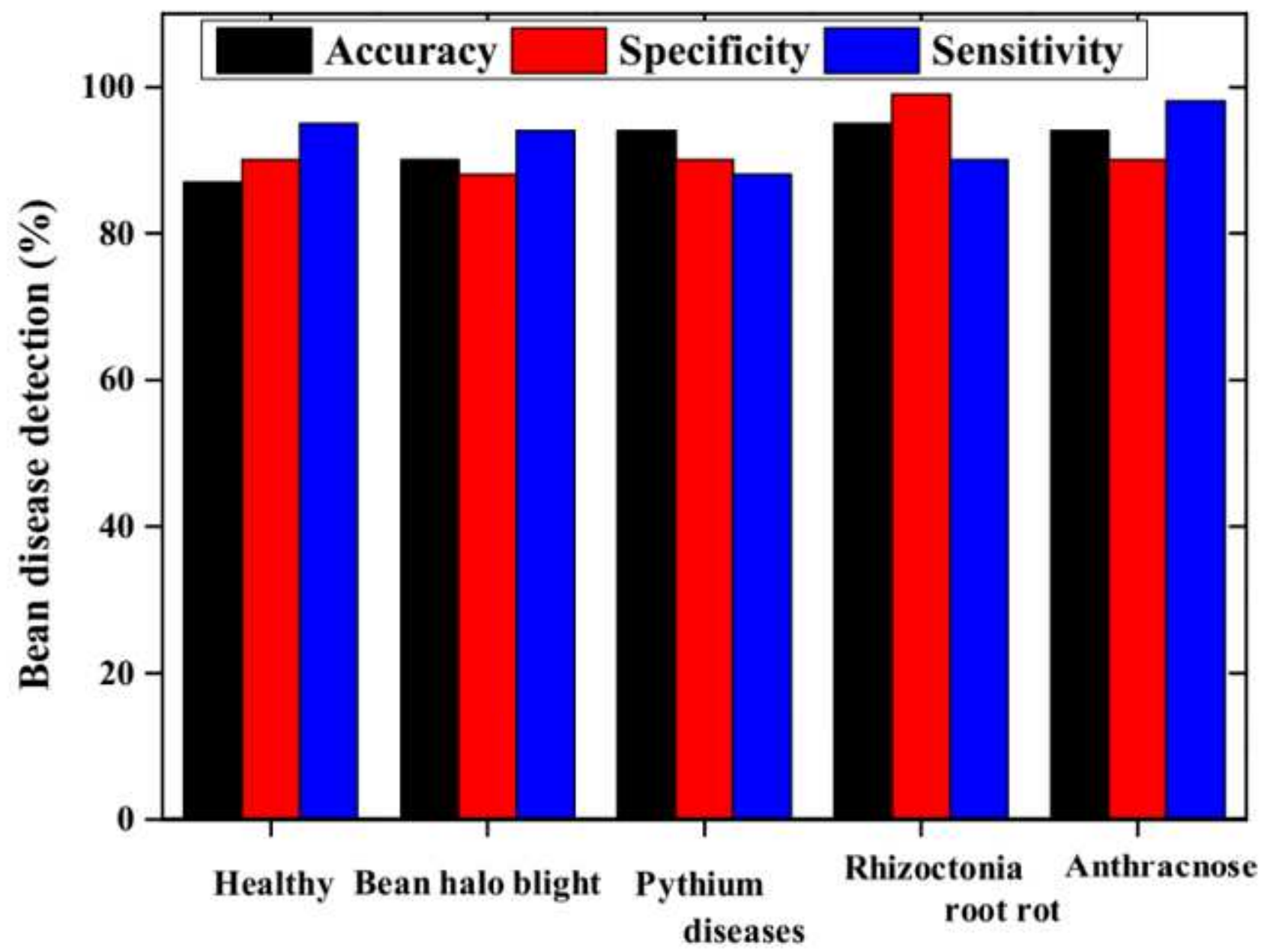

Figure 5

Proposed HDL-AOA performance analysis with respect to each class

\begin{tabular}{|c|c|c|c|c|c|}
\hline \multirow[b]{2}{*}{ Healthy } & Healthy & $\begin{array}{c}\text { Bean halo } \\
\text { blight }\end{array}$ & Pythium & $\begin{array}{c}\text { Rhizoctonia } \\
\text { root rot }\end{array}$ & Anthracnose \\
\hline & $84 \%$ & $4.5 \%$ & $8.12 \%$ & $2.12 \%$ & $1.26 \%$ \\
\hline \multirow{4}{*}{$\begin{array}{r}\text { Bean halo blight } \\
\text { Pythium diseases } \\
\text { hizoctonia root rot } \\
\text { Anthracnose }\end{array}$} & $0 \%$ & $89 \%$ & $2.68 \%$ & $3.56 \%$ & $4.76 \%$ \\
\hline & $4.15 \%$ & $1.66 \%$ & $91 \%$ & $3.19 \%$ & $0 \%$ \\
\hline & $4.65 \%$ & $0 \%$ & $3.89 \%$ & $91 \%$ & $0.46 \%$ \\
\hline & $3.78 \%$ & $1.12 \%$ & $3.01 \%$ & $0 \%$ & $92 \%$ \\
\hline
\end{tabular}

Figure 6

Confusion matrix with respect to each class 


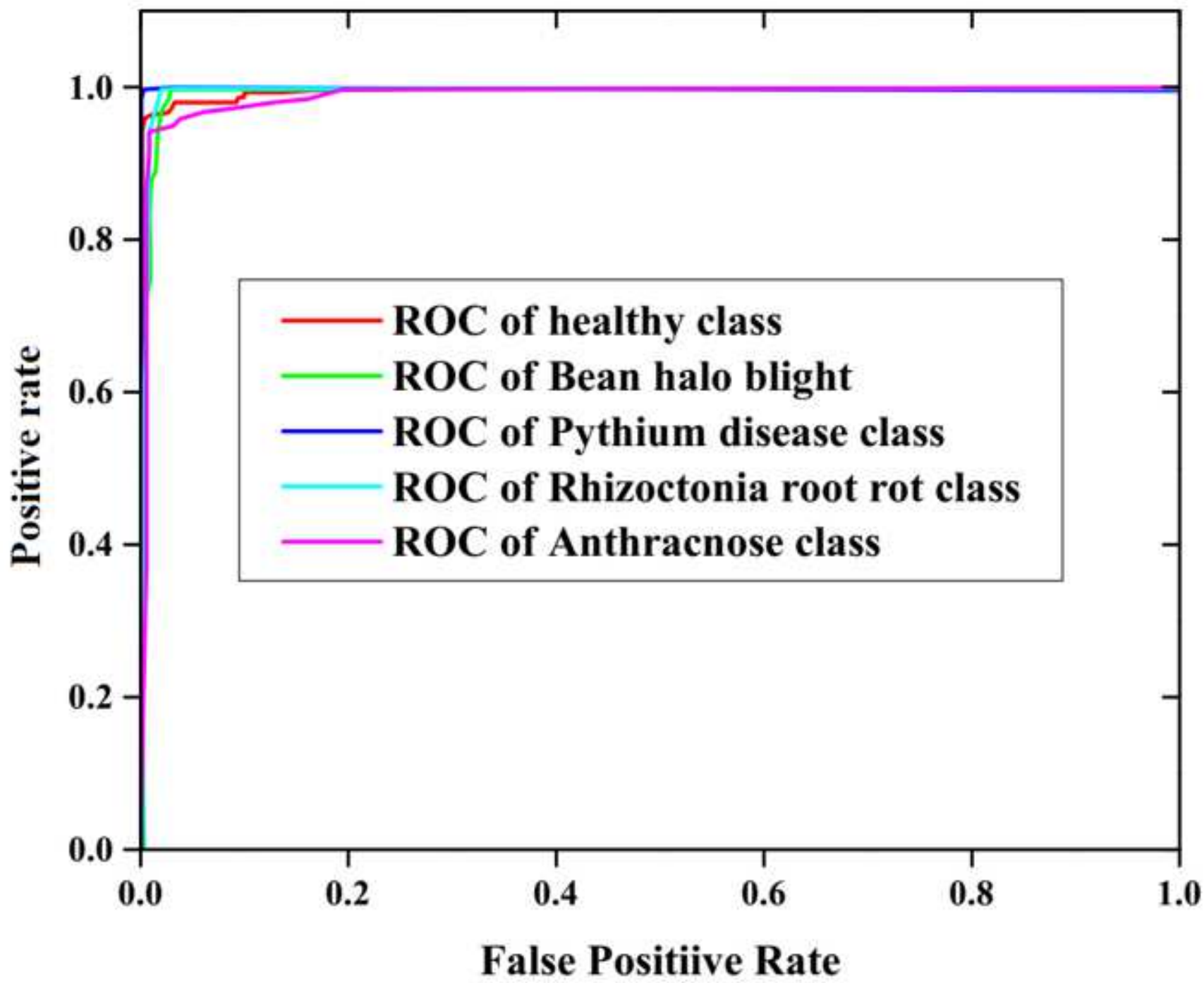

Figure 7

Proposed HDL-AOA performance analysis with respect to ROC 


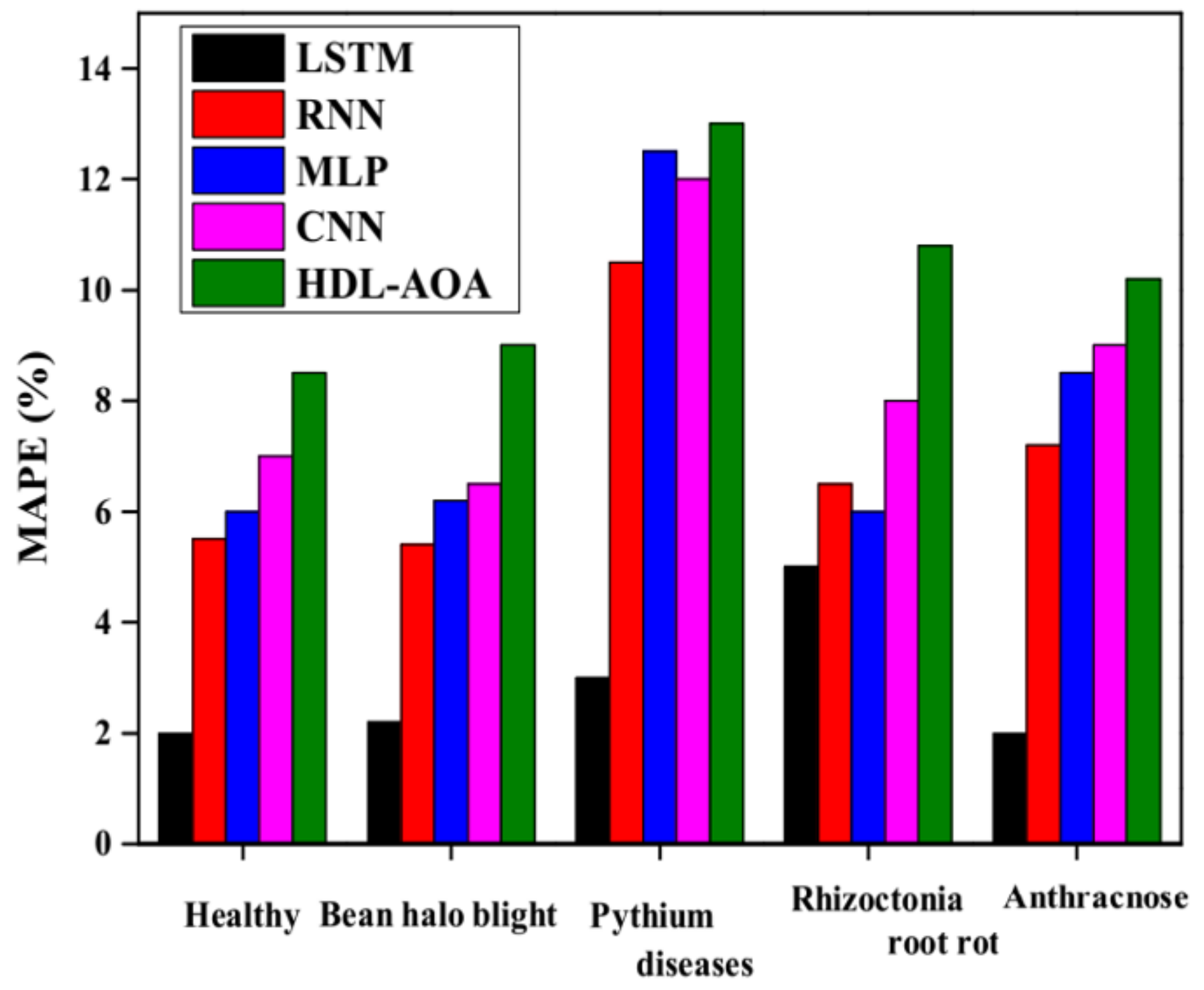

Figure 8

Performance evaluation of MAPE with respect to various methods and each class 